\title{
Optical Manipulation of Matter-Waves
}

\author{
R. Carretero-González ${ }^{a}$, P.G. Kevrekidis ${ }^{b}$, D.J. Frantzeskakis ${ }^{c}$, and B.A. Malomed ${ }^{d}$ \\ ${ }^{a}$ Nonlinear Dynamical Systems Group ${ }^{\dagger}$, Department of Mathematics, and Computational \\ Science Research Center ${ }^{\ddagger}$, San Diego State University, San Diego CA, 92182, USA; \\ ${ }^{b}$ Dept. of Mathematics and Statistics, University of Massachusetts, Amherst MA 01003, USA; \\ ${ }^{c}$ Dept. of Physics, University of Athens, Panepistimiopolis, Zografos, Athens 15784, Greece; \\ ${ }^{d}$ Dept. of Interdisciplinary Studies, School of Electrical Engineering, Faculty of Engineering, \\ Tel Aviv University, Tel Aviv 69978, Israel.
}

\begin{abstract}
Recent experimental and theoretical progress in the studies of Bose-Einstein condensation (BEC) has precipitated an intense effort to understand and control interactions of nonlinear matter-waves. Key ingredients in manipulations of matter-waves in BECs are a) external localized impurities (generated by focused laser beams) and $\mathrm{b}$ ) periodic potentials (generated by interference patterns from multiple laser beams illuminating the condensate). In this work we demonstrate the ability of time-dependent external optical potentials to drag, capture and pin a wide range of localized BEC states, such as dark and bright solitons. The stability and existence of pinned states is analyzed using perturbation techniques, which predict results that are well corroborated by direct numerical simulations. The control of these macroscopic quantum states has important applications in the realm of quantum storage and processing of information, with potential implications for the design of quantum computers.
\end{abstract}

Keywords: Matter waves, solitons, optical manipulation, Bose-Einstein condensation, nonlinear waves, optical lattices

\section{INTRODUCTION}

Since the first experimental realization of Bose-Einstein condensation (BEC) some 10 years ago, ${ }^{1}$ there has been a new surge of theoretical and experimental work on matter-waves and their nonlinear excitations. ${ }^{2}$ BECs were predicted by Bose and Einstein in the 1920's, ${ }^{3}$ who demonstrated that, at zero temperature, all atoms in a boson gas must fall into a single ground state. After their experimental creation became possible, BECs have been observed in dilute vapors of rubidium, sodium, lithium, and other alkali atoms. ${ }^{1}$ Presently, some forty experimental groups around the world routinely create BECs. The BECs production technology relies on laser techniques allowing to cool alkali atoms to temperatures below 1 nano-Kelvin, and confine them in traps built of magnetic and/or optical fields. ${ }^{2}$ These achievements were acknowledged through the 2001 Nobel prize in Physics. $^{4}$

Recent experimental advances in BECs allow for the creation and manipulation of matter-waves in diverse configurations and dimensionalities. In quasi-one dimensional (1D) settings, dark ${ }^{5}$ and bright ${ }^{6}$ solitons have $^{2}$ been observed in experiments with, respectively, repulsive and attractive atoms in BECs. Two-dimensional (2D) excitations, such as vortices ${ }^{7}$ and vortex lattices ${ }^{8}$ (see also the recent review of Ref. 9), have also been considered and realized experimentally. Bright solitons of the gap type in repulsive condensates have been predicted theoretically ${ }^{10}$ and have been created experimentally. ${ }^{11}$ Furthermore, stable multi-dimensional solitons supported by optical lattices have also been proposed. ${ }^{10,12,13}$

The creation and manipulation of matter-waves may have important and profound applications for quantum storage and processing of information. Particularly, solitons have the potential of being guided similarly to the

\footnotetext{
Further author information: (Send correspondence to R.C.G.)

R.C.G.: URL: http://rohan.sdsu.edu/ rcarrete/

${ }^{\dagger}$ URL: http://nlds.sdsu.edu/

${ }^{\ddagger}$ URL: http://www.csrc.sdsu.edu/csrc/
}

Optical Trapping and Optical Micromanipulation II, edited by Kishan Dholakia, Gabriel C. Spalding Proceedings of SPIE Vol. 5930 (SPIE, Bellingham, WA, 2005) - 0277-786X/05/\$15 - doi: 10.1117/12.615640 
manipulation of entire BECs in magnetic waveguides ${ }^{14}$ and atom chips. ${ }^{15}$ Furthermore, the similarity on the underlying dynamical equations between matter-waves and light suggests exciting possibilities to apply known results and techniques for optical solitons ${ }^{16}$ and quantum atom optics. ${ }^{17}$ The key ingredient in the manipulation of solitary matter-waves relies on the precise control of external optical potentials, created by one or multiple laser beams illuminating the BEC. For example, a sharply focused laser beam may be used to sculpture a desired BEC density distribution. ${ }^{5}$ Furthermore, depending on the detuning of the laser with respect to the frequency of a relevant intrinsic transition in the atom, this sharply focused laser beam can create a repulsive or attractive defect ("impurity"). The defect can be then used to pin or transport (drag) dark and bright solitons. Also, this impurity, when moving through the condensate at sufficient speed, can generate nonlinear excitations, such as solitons, vortices and periodic trains thereof. ${ }^{18-20}$

A particularly interesting possibility in BECs is engineering a periodic potential in the form of an optical lattice (OL), which is created as a standing-wave interference pattern between mutually coherent laser beams. ${ }^{21-26}$ The possibility to control the OL has led to the realization of many interesting phenomena, including Bloch oscillations, ${ }^{23,27}$ Landau-Zener tunneling ${ }^{21}$ (in the presence of an additional linear external potential), and classical $^{28}$ and quantum ${ }^{26}$ superfluid-insulator transitions. Recent reviews on the studies of nonlinear matterwaves trapped in OLs can be found in Refs. 29 and 30.

In this work we summarize some of our recent results concerning the generation and manipulation of dark and bright solitons by optical tools. In particular, we focus on: (a) the generation of soliton trains by a moving laser beam, ${ }^{20}$ (b) trapping bright solitons using a static laser beam, ${ }^{31}$ and (c) controlling the motion of dark ${ }^{32}$ and bright ${ }^{33}$ solitons with OLs. The paper is organized as follows. In Sec. 2, after introducing the model, we demonstrate the possibility of creating dark soliton trains by a moving impurity (induced by a focused laser beam). In Sec. 3 we examine the dynamics of bright solitons in the presence of attractive and repulsive localized static laser beams. In Sec. 4 we demonstrate that the motion of dark solitons can be controlled by means of the periodic OL. In Sec. 5 we investigate static and dynamical properties of bright solitons, and the possibility to manipulate them by an external time-dependent OL. Finally, we summarize our results and discuss possible generalizations in Sec. 6.

\section{NUCLEATION OF DARK SOLITONS}

In the framework of the mean-field theory, and at sufficiently low temperatures, the BEC is described by the Gross-Pitaevskii equation (GPE), ${ }^{34,35}$ that in normalized form reads ${ }^{36}$ :

$$
i u_{t}=-\frac{1}{2} \nabla^{2} u \pm|u|^{2} u+V(\mathbf{r}) u,
$$

where $u(\mathbf{r}, t)$ is to the mean-field wave function $\left(|u|^{2}\right.$ is the density of atoms), the ( \pm ) sign corresponds to repulsive $(+)$ and attractive $(-)$ interatomic interactions, and the external potential $V(\mathbf{r})$ accounts for the parabolic magnetic trap (the one which usually confines the condensate), or any other external potential (such as the OL, impurities, etc.). One of the most attractive features of the potential $V$ is the relative experimental ease of creating any desired external profile from laser interference patterns. The shape of the magnetic trap plays an important role determining the effective spatial dimensionality of the BEC. In experiments, highly anisotropic traps, that can be realized by a proper choice of strengths of different components of the magnetic fields, can be used to create either "pancake" -shaped condensates or "cigar" -shaped ones, which are, essentially, two- and one-dimensional objects; in the latter case, the BEC dynamics is accurately described by a one-dimensional (1D) GPE. ${ }^{37-39}$

In the present study we will concentrate on 1D BECs, described by the 1D version of Eq. (1), and we consider an external potential of the form

$$
\begin{aligned}
& V(x)=V_{\mathrm{MT}}(x)+V_{\mathrm{OL}}(x)+V_{\mathrm{Imp}}(x) \\
& \equiv \frac{1}{2} \Omega^{2} x^{2}+V_{\mathrm{OL}}^{(0)} \cos ^{2}(k x+\phi)+V_{\mathrm{Imp}}^{(0)} f(x-\xi) .
\end{aligned}
$$

The first potential term, $V_{\mathrm{MT}}$, accounts for the above-mentioned magnetic trap where the condensate is held, with trap frequency $\Omega$. The second term, $V_{\mathrm{OL}}$, represents the periodic potential generated by the OL with strength 

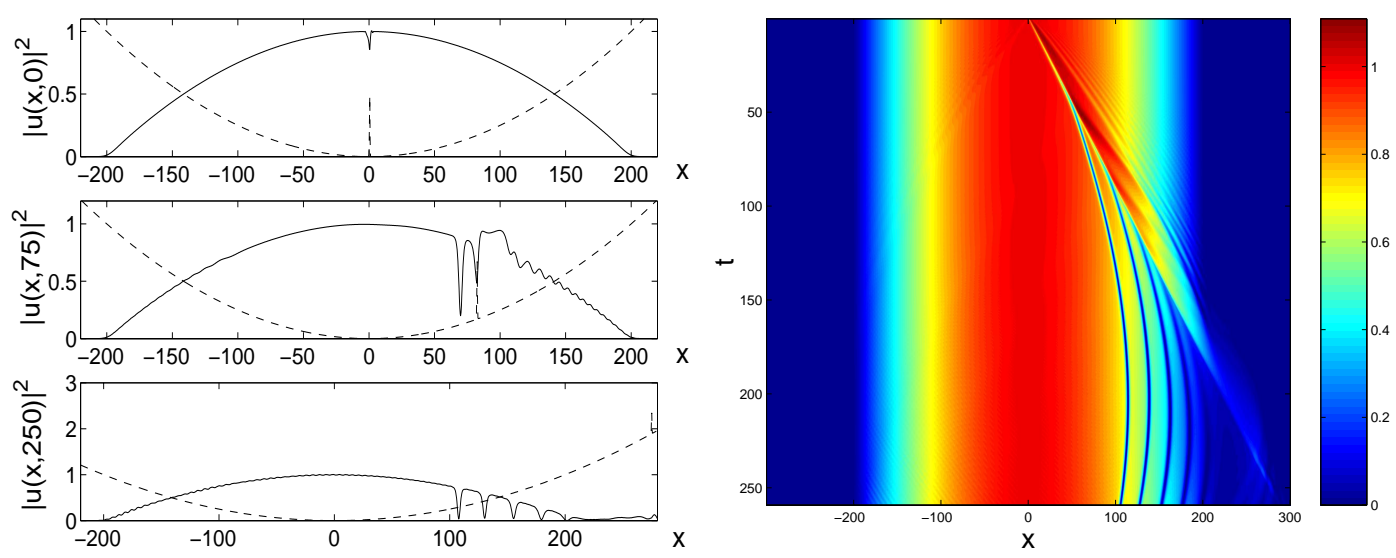

Figure 1. Typical scenario for the nucleation of dark solitons by an impurity moving (to the right) in a repulsive BEC. The parameter values for this run are $\Omega=\sqrt{2} / 200, V_{\operatorname{Imp}}^{(0)}=0.5, \epsilon=0.3$ and $v=1$. The left panels show the spatial profiles of the density (solid line) and the potential (dashed line) for $t=0,75,250$ while the right panel shows a contour plot of the spatio-temporal evolution of the atomic density.

$V_{\mathrm{OL}}^{(0)}$, wave number $k$ and detuning factor $\phi$. The wavenumber $k$ can be controlled by varying the angle $\theta$ between the counter-propagating laser beams at a given optical wavelength $\lambda_{\text {laser }}$, so that $\lambda \equiv 2 \pi / k=\left(\lambda_{\text {laser }} / 2\right) \sin (\theta / 2){ }^{40}$ Finally, the third term, $V_{\text {Imp }}$, represents a localized impurity with strength $V_{\text {Imp }}^{(0)}$ and profile $f$ located at position $\xi$, generated by a focused laser beam. Within the normalization adopted in Eq. (1), ${ }^{29} x$ is measured in units of the healing length $\xi_{h l}=\hbar / \sqrt{n_{0} g_{1 D} m}$, where $n_{0}$ is the peak density and $g_{1 D} \equiv g_{3 D} /\left(2 \pi l_{\perp}^{2}\right)$ is the effective 1D interaction strength, obtained upon integrating the 3D interaction strength $g_{3 D}=4 \pi \hbar^{2} a / m$ in the transverse directions ( $a$ is the scattering length, $m$ the atomic mass, and $l_{\perp}=\sqrt{\hbar / m \omega_{\perp}}$ is the transverse harmonic oscillator length, with $\omega_{\perp}$ being the transverse-confinement frequency). Additionally, $t$ is measured in units of $\xi_{h l} / c_{S}$ (where $c_{S}=\sqrt{n_{0} g_{1 D} / m}$ is the Bogoliubov speed of sound), the atomic density is rescaled by dividing it by the peak density $n_{0}$, and energy is measured in units of the chemical potential of the system, $\mu=g_{1 D} n_{0}$. It is important to stress that the GPE (1) (which is a variant of the commonly known nonlinear Schrödinger equation) models, in the context of optics, the propagation of pulses in optical fibers, or beams in waveguides and bulk media. In the former case, time $t$ and space $x$ represent the spatial coordinate along the fiber and reduced time, while, in the latter case, they represent the longitudinal and transverse coordinates, respectively. In both cases, $|u|^{2}$ represents the light intensity, and the nonlinear term represents the response of a defocusing $(+)$ or focusing $(-)$ nonlinear medium.

In the absence of the OL, a moving impurity with $\xi=v t$ is capable of nucleating dark solitons inside a repulsive BEC. ${ }^{20}$ To model the localized impurity we use the most natural beam shape, i.e., the Gaussian profile

$$
V_{\text {Imp }}(x, t)=V_{\text {Imp }}^{(0)} \exp \left(-(x-v t)^{2} / \epsilon^{2}\right)
$$

with the amplitude $V_{\text {Imp }}^{(0)}$, width $\epsilon$ and velocity $v>0$. It is known that an impurity moving across a uniform background at a speed larger than the Bogoliubov speed of sound $c_{S}$ (in our units $c_{S}=\sqrt{2}$ ) will generate sound waves. ${ }^{18,19}$ Since the background density of the stationary condensate is inhomogeneous (due to the presence of the magnetic trap) and decays to zero away from its center, there will always be a point beyond which the density is small enough for the impurity with a given velocity to become supersonic. Therefore, emission of waves should occur for any speed $v$ of the impurity. In Fig. 1 we show a typical example of a localized impurity moving through the condensate. It is clearly seen that a train of dark solitons is indeed generated by the impurity as it traverses the condensate. In Ref. 20, we showed that the dependence of the critical density of the nucleation on the impurity speed $v$ features two different regimes: a) for small $v$ one can assume adiabaticity, and it is possible to approximate the critical nucleation density using a perturbative approach ${ }^{19}$; b) for larger $v$, this adiabatic approach is no longer valid and the critical nucleation density is always smaller than in the former regime. Another important feature that can be noticed from Fig. 1 is that after the first nucleation, a series 

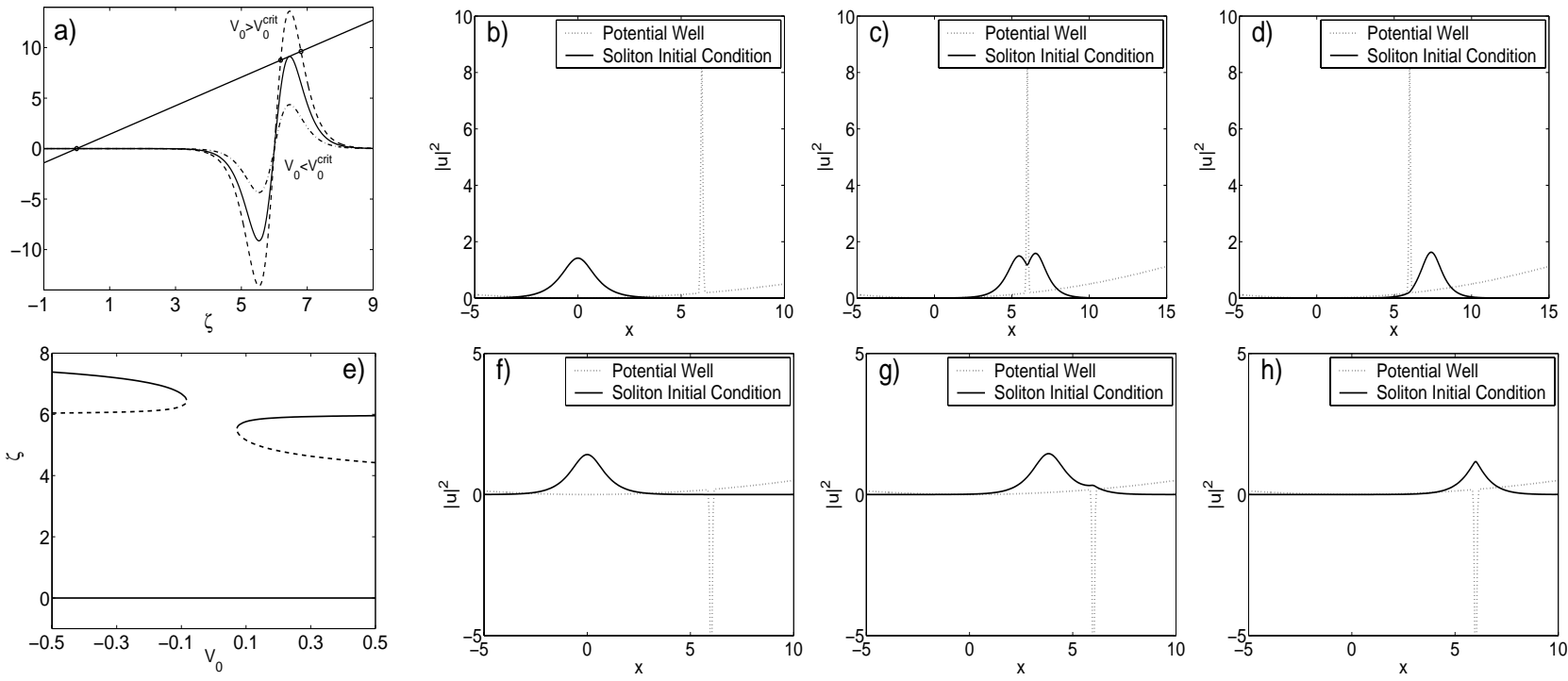

Figure 2. a) and d) Saddle-node bifurcation and stability for stationary states of a bright soliton pinned by a localized impurity. The panels in b)-d) and f)-h) represent, respectively, all the steady states for a repulsive and an attractive impurity of strength $V_{\text {Imp }}^{(0)}=\mp 1$. The relevant parameters for all panels correspond to a magnetic trap with strength $\Omega=0.1$ and an impurity located at $\xi=6$

of periodic nucleations occur, giving rise to a quasi-equispaced train of dark solitons. Further investigation ${ }^{20}$ reveals that the distance between solitons in the chain decays monotonically with the increase of the impurity speed $v$ (results not shown here).

\section{BRIGHT SOLITONS PINNED BY LOCALIZED IMPURITIES}

In this section we study the equilibrium positions and their corresponding stability characteristics for bright solitons in the presence of a focused laser beam. Therefore, we assume the attractive nonlinearity in the GPE (1) which, in the absence of the external potential $(V=0)$, supports bright soliton solutions of the form

$$
u_{s}=\eta \operatorname{sech}[\eta(x-\zeta)] \exp \left(i \eta^{2} t / 2\right)
$$

where $\eta$ is the soliton's amplitude, and $\zeta$ is the coordinate of its center. Moving solitons can be generated from the zero-velocity one by the Galilean boost. The persistence and dynamics of the bright solitary waves in the presence of the potential $V(x)$ can be studied by means of the standard perturbation theory (see e.g., Refs. 41 and 42 or through the Lyapunov-Schmidt reduction techniques used in Ref. 43). Let us consider bright solitons (in the absence of the OL, i.e. $V_{\mathrm{OL}}^{(0)}=0$ ), which are present in a potential consisting of the combination of a magnetic trap $V_{\mathrm{MT}}$ and an infinitely localized impurity of the form

$$
V_{\operatorname{Imp}}(x, t)=-V_{\operatorname{Imp}}^{(0)} \delta(x-\xi) .
$$

Treating the soliton as a quasi-particle, it can readily be found that the impurity and the magnetic trap give rise to effective potential forces acting on the particle, thus inducing a Newton-type dynamics for its center of mass $\zeta(t),{ }^{31}$

$$
\begin{aligned}
& \ddot{\zeta}=\quad F_{\mathrm{I}}+F_{\mathrm{MT}} \\
& \equiv 2 \eta^{3} V_{\text {Imp }}^{(0)} \tanh (\eta(\xi-\zeta)) \operatorname{sech}^{2}(\eta(\xi-\zeta))-2 \Omega^{2} \zeta \eta .
\end{aligned}
$$

The equation of motion for the soliton's center (6) has, generically, one or three fixed points, depending on the parameters (see Fig. 2.a). Upon further inspection, ${ }^{31}$ the trivial fixed point $\zeta=0$, corresponding to a bright soliton at the bottom of the magnetic trap, is stable. As the strength of the impurity $V_{\operatorname{Imp}}^{(0)}$ is increased, fixed 

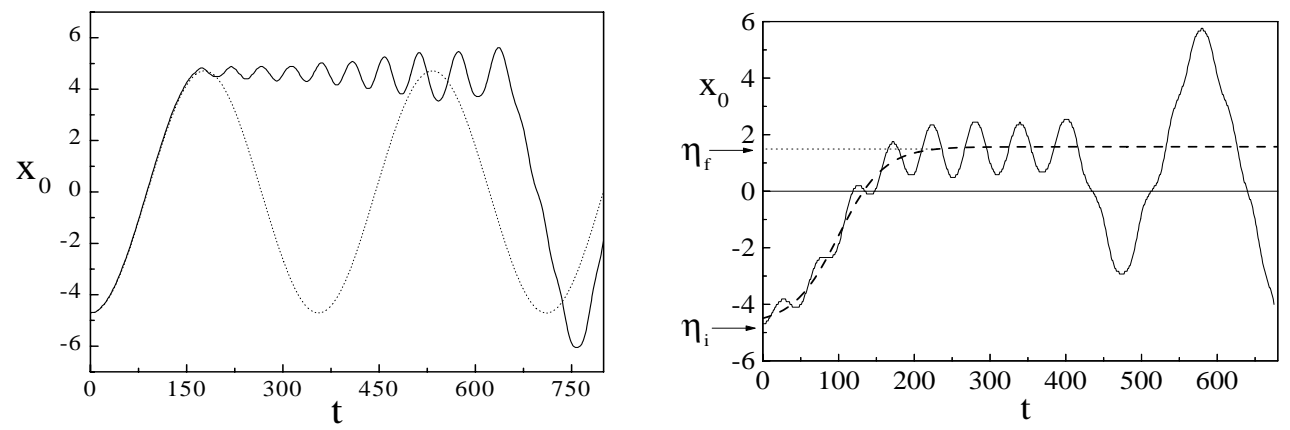

Figure 3. Left: Entrapment of a moving dark soliton by a potential well belonging to an optical lattice switched on at $t_{0}=177$ (and $T=5$ ). The dotted/solid line corresponds to the dark soliton's trajectory in the absence/presence of the time-modulated optical lattice. Right: The targeted transfer of a dark soliton. The continuous and dashed curves show, respectively, the actual law of motion of the trapped dark soliton, and that of the driving optical lattice drive (10), with $t_{0}=100$ and $T=60$. In both panels, the strength of the parabolic trap is $\Omega=0.025$, and parameters of the optical lattice are $V_{\mathrm{OL}}^{(0)}=0.07$ and $k=1$.

points of Eq. (6) undergo a saddle-node bifurcation which creates two new steady states near the center of the impurity, see Fig. 2.e). For the repulsive impurity $\left(V_{\mathrm{Imp}}^{(0)}<0\right)$, the fixed point closest to the center of the impurity is unstable, while the fixed point to the right of the impurity is stable. For the attractive impurity $\left(V_{\operatorname{Imp}}^{(0)}>0\right)$, the stability is reversed and the stable fixed point is the one closest to the impurity. In Fig. 2 we depict examples of all the fixed points found for this system. Panels b) through d) correspond to the repulsive impurity, while panels f) through $\mathrm{h}$ ) correspond to the attractive one.

These results are relevant to the trapping and, potentially, to the guidance of bright matter-wave solitons. They illustrate the possibility of pinning bright solitons at desired locations, using an appropriate localized impurity created by a focused laser beam. The robustness of some of the fixed points paves the way for potentially dragging bright solitons in a controllable way by means of an adiabatically moving impurity. An important result in that connection is that the beam's intensity must exceed a critical value in order to pin (and potentially carry) the soliton. ${ }^{33}$

\section{DRIVING DARK SOLITONS BY OPTICAL LATTICES}

We will now consider the motion of dark solitons trapped in a combined magnetic and OL potential of the form (2), without the impurity $\left(V_{\operatorname{Imp}}^{(0)}=0\right)$. In the absence of any potential $(V=0)$, the repulsive GPE (1) possesses exact dark soliton solutions of the form ${ }^{44}$ :

$$
u(x, t)=u_{0}(\cos \varphi \tanh \zeta+i \sin \varphi) \exp \left(-i \mu_{0} t\right),
$$

where $u_{0}$ is the amplitude of the uniform background, $\mu_{0} \equiv u_{0}^{2}$ corresponds to the dimensionless chemical potential (in the present work we take $\mu_{0}=1$ ), $\varphi$ is the phase shift across the dark soliton $(|\varphi|<\pi / 2$ ), and $\zeta \equiv u_{0}(\cos \varphi)\left[x-u_{0}(\sin \varphi) t\right]$. The amplitude (depth) and velocity of the dark soliton are $u_{0} \cos \varphi$ and $u_{0} \sin \varphi$, respectively. The limiting case $\varphi=0$ corresponds to a quiescent black soliton, $u=u_{0} \tanh \left(u_{0} x\right) \exp \left(-i \mu_{0} t\right)$. Many theoretical studies have been devoted to the dynamics of dark solitons in the presence of external potentials. ${ }^{45,46}$ In the presence of the magnetic trap only, the dark soliton oscillates with the frequency $\omega_{f}=\Omega / \sqrt{2}$. In the presence of both the magnetic trap and the OL, the motion of the dark soliton depends on the OL's period, as compared to the soliton's width. ${ }^{47}$ The long-period OL can be treated as a perturbation, and the dynamics of the dark soliton can adjust itself to the smooth potential. ${ }^{47}$ For the short-period OL, a multiple-scale expansion reveals ${ }^{47}$ that the system reduces to the motion of a dark soliton inside an effective magnetic trap (with no OL) with a renormalized strength, in which the oscillation frequency is $\omega_{\text {eff }}=\omega_{f}\left(1-\left(7 \delta^{2}\right) /\left(256 k^{2}\right)\right)$, where $\delta=V_{\mathrm{OL}}^{(0)} / k$.

Let us now consider in more detail the effects of a time modulated OL of the form

$$
V_{\mathrm{OL}}(x, t)=g(t) V_{\mathrm{OL}}^{(0)} \cos ^{2}[k(x-y(t))+\phi],
$$


where $g(t)$ is a switching function and $y(t)$ is the time-varying position of the OL. First, we try to capture (freeze) the motion of a dark soliton that is freely oscillating inside the magnetic trap $\left(V_{\mathrm{OL}}^{(0)}=0\right)$. For that purpose we set up a dark soliton performing harmonic oscillation between $x=-3 \pi / 2$ and $x=+3 \pi / 2$. Then we switch on a static OL $(y(t)=0)$, using the smooth switching function

$$
g(t)=\frac{1}{2}\left[1+\tanh \left(\frac{t-t_{0}}{T}\right)\right]
$$

with $t_{0}=177$ corresponding to the time when the dark soliton is at its maximum position $(x=3 \pi / 2)$ and with zero velocity. A typical example of the resulting dynamics is depicted in the left panel of Fig. 3. As the OL is switched on, the dark soliton finds itself inside a local minimum of the OL where it performs small oscillations that gradually increase due to weak radiation loss. ${ }^{48,49}$ As seen from the figure, the dark soliton remains trapped for relatively long times and then eventually escapes due to the radiation loss. More detailed simulations also demonstrate that the dark soliton can be trapped at any time and at any position, as long as the switching-on time $T$ is small enough and the OL strength in sufficiently large.

We now consider a challenging possibility of transferring a dark soliton by steadily moving the OL. For that purpose, we start with a dark soliton already trapped at an OL minimum and then we proceed by time-varying the position of the OL (with $g(t)=1)$ :

$$
y(t)=\eta_{i}+\frac{1}{2}\left(\eta_{f}-\eta_{i}\right)\left[1+\tanh \left(\frac{t-t_{0}}{T}\right)\right],
$$

where $\eta_{i}$ and $\eta_{f}$ are the initial and (desired) final positions of the dark soliton's center, while $T$ and $t_{0}$ are constants controlling, respectively, the duration and the beginning of the transfer. Extensive simulations demonstrate the possibility of the controlled transfer of dark solitons by moving OLs. A typical example is shown in the right panel of Fig. 3, where a soliton initially placed at $\eta_{i}=-3 \pi / 2$ is delivered to $\eta_{f}=\pi / 2$. As in the previous example, radiation loss is again responsible for a gradual increase in the amplitude of local oscillations that eventually leads to the escape of the dark soliton from the relevant potential well.

\section{DRIVING BRIGHT SOLITONS BY OPTICAL LATTICES}

In this section we examine manipulations of bright solitons by OLs. We thus use the GPE model (1) with the attractive nonlinearity and we apply the same OL potential (8) as defined in the previous section. Using e.g. $\phi=-\pi / 2$ and the same switching function (9) makes it possible to steadily move the OL as per Eq. (10). For a static OL $(g(t)=1$ and $y(t)=0)$, there exist several possible steady states generated by the competition of the magnetic trap and the OL. As in Sec. 3, it is possible to study the dynamics of the bright soliton using standard perturbation techniques. ${ }^{41,42}$ A careful analysis ${ }^{33}$ shows that the bright soliton behaves like a quasi-particle inside an effective potential

$$
V_{\text {eff }}(x)=\eta \Omega^{2} x^{2}-\pi V_{\mathrm{OL}}^{(0)} k \cos (2 k x) \operatorname{csch}(k \pi / \eta) .
$$

Notice that, depending on values of the parameters, this potential may have a single extremum at $x=0$, or multiple ones. The stability of the soliton resting at a stationary position can also be analyzed in terms of the effective potential (11): the position is stable if it corresponds to a potential minimum. This well-known result can be rigorously derived using the theory developed in Ref. 50 and reformulated in Ref. 51 (see also Refs. 52 and 53). In particular, the curvature of the effective potential at the stationary position determines the stability of the fixed point: minima and maxima of the effective potential (11) give rise, respectively, to stable and unstable equilibria. Extensive numerical simulations (not shown here) corroborate this expectation. ${ }^{33}$ Note that minima of the effective potential (11) differ from the minima of the external potential $V(x)$ which traps the atoms.

After addressing the stability of bright solitons at different local minima of the potential, let us now study the possibility of their manipulation by steadily translating the OL. As in the previous section, we try two different approaches. The first approach consists of snaring a bright soliton performing harmonic oscillations inside the magnetic trap by suddenly switching on the OL, using the switching-on function $g(t)$ [cf. Eq. (9)]. The second approach consists of adiabatically transferring a bright soliton by steadily moving the OL using the translation 

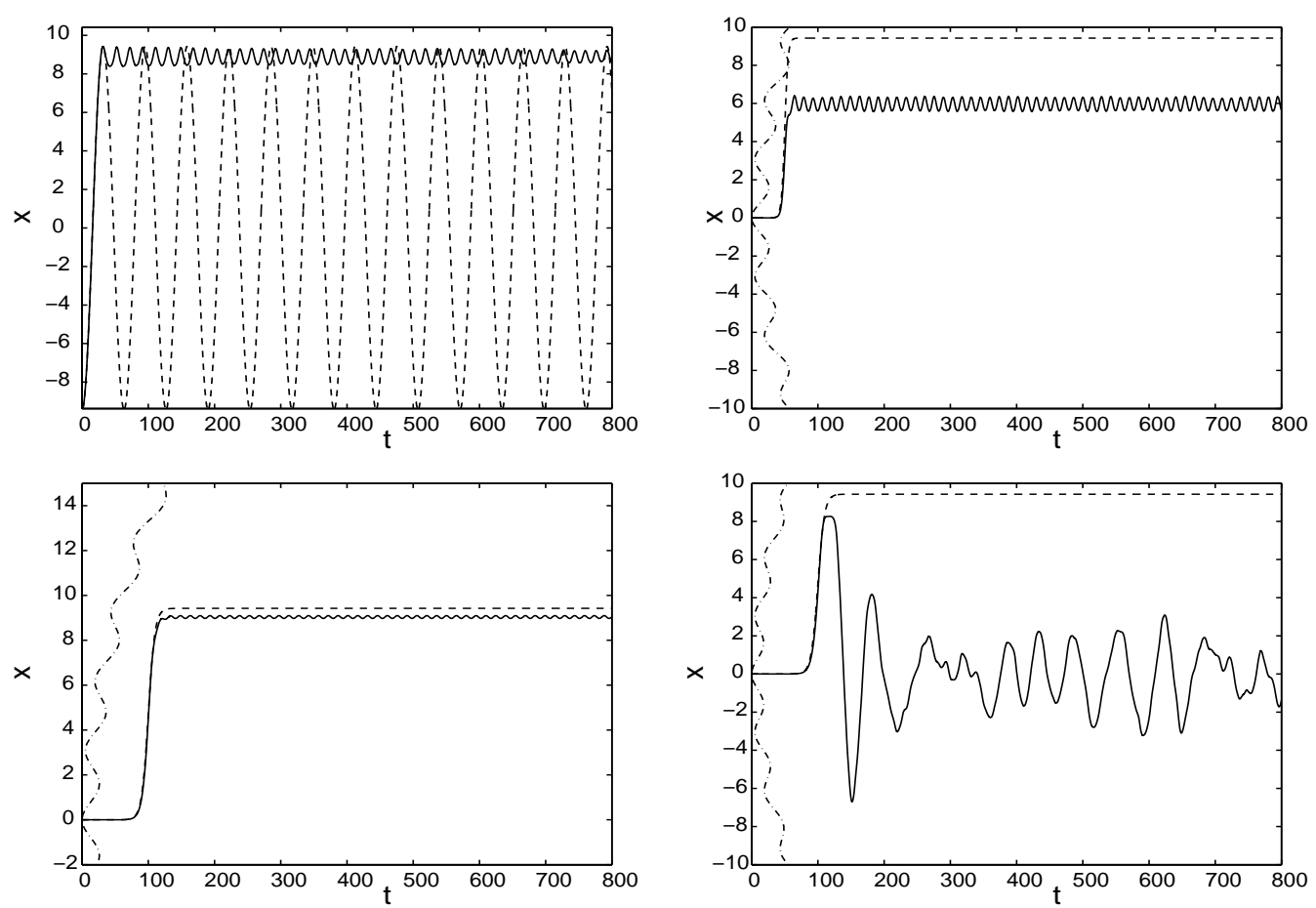

Figure 4. Manipulating bright solitons by time-dependent optical lattices. Top-left: An example of snaring the originally moving soliton using the optical lattice. The panel shows the motion of the soliton's center of mass (solid line). The dashed line shows the situation without the OL (but in the presence of the magnetic trap). Top-right and bottom-left: transferring a bright soliton by means of a slowly moving OL. The center of mass of the soliton is depicted by the solid line while the motion of the OL is depicted by the dashed line. The parameters for these two panels are, respectively, $\left(t_{0}, T\right)=(50,5)$ and $\left(t_{0}, T\right)=(100,10)$, both with $V_{0}=0.25$. Bottom-right: A shallower OL, $V_{0}=0.17$, is not capable of snaring the soliton.

law (10). Numerical simulations show that, for sufficiently large OL strength $V_{\mathrm{OL}}^{(0)}$, it is possible to snare and transport the bright soliton essentially at will. ${ }^{33}$ Typical situations are depicted in Fig. 4 . The top-left panel depicts a situation similar to the left panel of Fig. 3 for a dark soliton. Namely, an oscillating bright soliton inside the magnetic trap is snared by the OL which is switched on at the precise moment when the soliton was at its maximum position. This position is very close to a minimum of the effective potential (11). As shown in this panel, the soliton is safely trapped by the OL where, in contrast with the dark soliton example of the previous section, it performs small oscillations indefinitely long. In fact, a key difference from the dark soliton case of the previous section and the current case of a bright soliton, is that radiation loss acts in opposite ways for the two types of solitons. Namely, for a dark soliton, radiation loss corresponds to a gain in velocity (as the dark soliton has an effective negative mass), while radiation loss tends to damp the movement of bright solitons. Therefore, once a bright soliton is trapped by a local minimum it will stay there indefinitely (provided that it is near a stable equilibrium point of the effective potential).

In the top-right and bottom-left panels of Fig. 4 we depict two examples of bright soliton transport using a steadily moving OL. Both panels represent the transport of the bright soliton using the same OL profile (with $g(t)=1$ ) but with different motion $y(t)$. In the first panel (top-right) we use $T=5$ while in the second panel (bottom-left) we use $T=10$. It is clear from the figure that, if the transport is not sufficiently slow to adiabatically drag the soliton to its final position, the soliton gets trapped in an intermediate OL well without reaching its destination. If, however, the drag is applied with a sufficient degree of adiabaticity, the bright soliton is safely delivered to the target well. The bottom-right panel in Fig. 4 presents an example when the OL lattice strength is not sufficiently large to properly transport the bright soliton. In this case the soliton is lost just before it is delivered to the third well of the OL potential and then performs complicated oscillations about the 

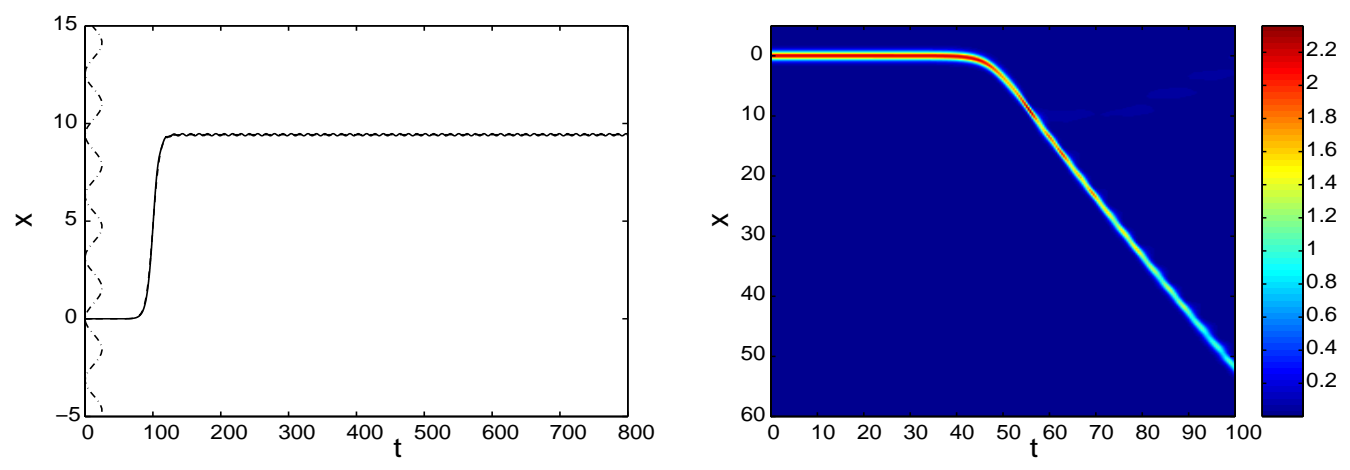

Figure 5. The same as the previous figure, but with $\Omega=0$ (i.e., in the absence of the magnetic trap). For $t_{0}=100$ and $T=10$ (left panel) the soliton is delivered to its final location of $x_{\text {fin }}=3 \pi$. However, the same is not true for $t_{0}=50$ and $T=5$ (right panel), where the soliton fails to stop but rather continues its motion, losing more and more of its power through emission of radiation.

center of the magnetic trap.

A similar numerical experiment in the absence of the magnetic trap $(\Omega=0)$ is shown in Fig. 5. The left panel depicts the successful transfer of the soliton by the OL with $V_{\mathrm{OL}}^{(0)}=0.25, g(t)=1$ and $\left(t_{0}, T\right)=(100,10)$. However, once again, the same experiment, if not performed with a sufficient degree of adiabaticity [cf. right panel of Fig. 5, with $\left.\left(t_{0}, T\right)=(50,5)\right]$, is not successful in transporting the soliton to the desired location. Instead, the soliton continues to move with an approximately constant speed, emitting radiation waves and decreasing its amplitude.

\section{CONCLUSION}

In this paper we have reviewed a number of scenarios involving the creation and control of matter-wave solitons through optical manipulation. We considered both dark and bright solitons in, respectively, repulsive and attractive one-dimensional Bose-Einstein condensates (BECs). The first scenario that we discussed pertains to the nucleation of dark solitons by a moving impurity created by a focused laser beam. We note that, due to the inhomogeneous shape of the condensate's density (decaying to zero away from its center), the impurity will always reach supersonic velocities and thus generate dark solitons. Furthermore, a series of solitons is created as the impurity continues to traverse the condensate, forming a train of quasi-equispaced solitons. We also examined the pinning of bright solitons in the presence of a focused laser beam. It is shown that, if the beam is strong enough, it can support nontrivial stable fixed points that depend of the beam's position. This opens the possibility to transport bright solitons by means of adiabatically translating the beam's position.

Finally, we studied the manipulation of dark and bright solitons by means of a periodic potential generated by an optical lattice (OL). We showed that dark and bright solitons may be manipulated, if the OL strength is sufficiently large, by a sufficiently adiabatic translation of the OL. The cardinal difference between dark and bright solitons is the fact that, due to radiation loses, dark solitons tend to increase their oscillation amplitudes within a local potential well, while bright solitons display damped oscillations. Therefore, dark solitons can only be trapped for a finite interval of time while bright solitons may be trapped indefinitely.

An interesting and potentially important generalization of the current study would be to extend it to the case of two- and, potentially, three-dimensional settings. Particularly interesting should be the study of optical manipulations of BEC vortices by means of optical tools. Also, given the recent prediction of solitons and vortices in multi-dimensional OL potentials ${ }^{10}$ (for recent experimental work on a similar topic in nonlinear optics, see Refs. 54 and 55 and references therein), it would be interesting too to implement similar dragging and manipulation of solitons in higher dimensions. The consideration of these scenarios is currently in progress and will be reported in future publications. 


\section{ACKNOWLEDGMENTS}

The work of B.A.M. was supported in a part by the Israel Science Foundation through the grant No. 8006/03. P.G.K. gratefully acknowledges support from NSF-DMS-0204585, NSF-CAREER. R.C.G. and P.G.K. also acknowledge support from NSF-DMS-0505663. D.J.F. acknowledges support from the Special Research Account of the University of Athens (grant No. 70/4/5844).

\section{REFERENCES}

1. M.H. Anderson, J.R. Ensher, M.R. Matthews, C.E. Wieman, and E.A. Cornell, Science 269, 198 (1995); K.B. Davis, M.-O. Mewes, M.R. Andrews, N.J. van Druten, D.S. Durfee, D.M. Kurn, and W. Ketterle Phys. Rev. Lett. 75, 3969 (1995);

C.C. Bradley, C.A. Sackett, J.J. Tollett, and R.G. Hulet Phys. Rev. Lett. 75, 1687 (1995).

2. F. Dalfovo, S. Giorgini, L. P. Pitaevskii, and S. Stringari, Rev. Mod. Phys. 71, 463 (1999);

A. J. Leggett, ibid. 73, 307 (2001);

E.A. Cornell and C.E. Wieman, ibid. 74, 875 (2002);

W. Ketterle, ibid. 74, 1131 (2002).

3. S.N. Bose, Z. Phys. 26178 (1924);

A. Einstein, Sitzber. Kgl. Preuss. Akad. Wiss. 261 (1924);

A. Einstein, Sitzber. Kgl. Preuss. Akad. Wiss. 3 (1925).

4. http://www.nobel.se/physics/laureates/2001/.

5. S. Burger, K. Bongs, S. Dettmer, W. Ertmer, K. Sengstock, A. Sanpera, G. V. Shlyapnikov, and M. Lewenstein, Phys. Rev. Lett. 83, 5198 (1999);

J. Denschlag, J. E. Simsarian, D. L. Feder, C. W. Clark, L. A. Collins, J. Cubizolles, L. Deng, E. W. Hagley, K. Helmerson, W. P. Reinhardt, S. L. Rolston, B. I. Schneider, and W. D. Phillips, Science 287, 97 (2000); B. P. Anderson, P. C. Haljan, C. A. Regal, D. L. Feder, L. A. Collins, C. W. Clark, and E. A. Cornell, Phys. Rev. Lett. 86, 2926 (2001).

6. K. E. Strecker, G. B. Partridge, A. G. Truscott, and R. G. Hulet, Nature 417, 150 (2002);

L. Khaykovich, F. Schreck, G. Ferrari, T. Bourdel, J. Cubizolles, L. D. Carr, Y. Castin, and C. Salomon, Science 296, 1290 (2002).

7. M. R. Matthews, B. P. Anderson, P. C. Haljan, D. S. Hall, C. E. Wieman, and E. A. Cornell, Phys. Rev. Lett. 83, 2498 (1999);

K. W. Madison, F. Chevy, W. Wohlleben, and J. Dalibard, Phys. Rev. Lett. 84, 806 (1999);

S. Inouye, S. Gupta, T. Rosenband, A. P. Chikkatur, A. Görlitz, T. L. Gustavson, A. E. Leanhardt, D. E. Pritchard, and W. Ketterle, Phys. Rev. Lett. 87, 080402 (2001).

8. J.R. Abo-Shaeer, C. Raman, J.M. Vogels, W. Ketterle, Science 292, 476 (2001);

J.R. Abo-Shaeer, C. Raman, W. Ketterle, Phys. Rev. Lett. 88, 070409 (2002);

P. Engels, I. Coddington, P.C. Haljan and E.A. Cornell, Phys. Rev. Lett. 89, 100403 (2002).

9. P.G. Kevrekidis, R. Carretero-González, D.J. Frantzeskakis and I.G. Kevrekidis, Mod. Phys. Lett. B 18, 1481 (2005).

10. B. B. Baizakov, V. V. Konotop, and M. Salerno, J. Phys. B 35, 5105 (2002);

E.A. Ostrovskaya and Yu.S. Kivshar, Phys. Rev. Lett. 90, 160407 (2003).

11. B. Eiermann, Th. Anker, M. Albiez, M. Taglieber, P. Treutlein, K.-P. Marzlin, and M.K. Oberthaler, Phys. Rev. Lett. 92, 230401 (2004).

12. B. B. Baizakov, B.A. Malomed, and M. Salerno, Europhys. Lett. 63, 642 (2003).

13. B. B. Baizakov, B.A. Malomed, and M. Salerno, in: Nonlinear Waves: Classical and Quantum Aspects, ed. by F.Kh. Abdullaev and V.V. Konotop, p. 61 (Kluwer Academic Publishers: Dordrecht, 2004); ibid. Multidimensional solitons in a low-dimensional periodic potential, Phys. Rev. A, in press.

14. A.E. Leanhardt, A.P. Chikkatur, D. Kielpinski, Y. Shin, T.L. Gustavson, W. Ketterle, and D.E. Pritchard, Phys. Rev. Lett. 89, 040401 (2002);

H. Ott, J. Fortagh, S. Kraft, A. Gunther, D. Komma, and C. Zimmermann, Phys. Rev. Lett. 91, 040402 (2003). 
15. W. Hänsel, P. Hommelhoff, T.W. Hansch, and J. Reichel, Nature 413, 498 (2001);

R. Folman and J. Schmiedmayer, Nature 413, 466 (2001);

J. Reichel, Appl. Phys. B 74, 469 (2002);

R. Folman, P. Krueger, J. Schmiedmayer, J. Denschlag and C. Henkel, Adv. Atom. Mol. Opt. Phys. 48, 263 (2002).

16. Yu.S. Kivshar and G.P. Agrawal, Optical Solitons: From Fibers to Photonic Crystals (Academic Press, San Diego, 2003).

17. K. Mølmer, New J. Phys. 5, 55 (2003).

18. T. Frisch, Y. Pomeau and S. Rica, Phys. Rev. Lett. 69, 1644 (1992);

T. Winiecki, J. F. McCann and C. S. Adams, Phys. Rev. Lett. 82, 5186 (1999);

B. Jackson, J. F. McCann and C. S. Adams, Phys. Rev. A 61, 013604 (2000);

G. E. Astracharchik, L.P. Pitaevskii, Phys. Rev. A 70, 013608 (2004);

C. Raman, M. Köhl, D. S. Durfee, C. E. Kuklewicz, Z. Hadzibabic and W. Ketterle, Phys. Rev. Lett. 83, $2502(1999)$;

R. Onofrio, C. Raman, J.M. Vogels, J. R. Abo-Shaeer, A.P. Chikkatur and W. Ketterle, Phys. Rev. Lett. 85, 2228 (2000);

P. Leboeuf and N. Pavloff, Phys. Rev. A 64, 033602 (2001);

N. Pavloff, Phys. Rev. A 66, 013610 (2002);

A. Radouani, Phys. Rev. A, 70, 013602 (2004);

G. Theocharis, P.G. Kevrekidis, H.E. Nistazakis, D.J. Frantzeskakis and A.R. Bishop, Phys. Lett. A 337, 441 (2005).

19. V. Hakim, Phys. Rev. E 55, 2835 (1997).

20. P.G. Kevrekidis, D.J. Frantzeskakis, R. Carretero-González, B.A. Malomed, S. Nandi, and A.R. Bishop. "Soliton trains and vortices as form of Cherenkov radiation in one- and two-dimensional trapped BoseEinstein condensates". In preparation.

21. B.A. Anderson and M.A. Kasevich, Science 282, 1686 (1998);

M. Jona-Lasinio, O. Morsch, M. Cristiani, N. Malossi, J. H. Müller, E. Courtade, M. Anderlini, and E. Arimondo, Phys. Rev. Lett. 91, 230406 (2003);

V.V. Konotop, P.G. Kevrekidis, and M. Salerno, arXiv:cond-mat/0404608.

22. C. Orzel, A.K. Tuchman, M.L. Fenselau, M. Yasuda, and M.A. Kasevich, Science 291, 2386 (2001).

23. O. Morsch, J.H. Muller, M. Cristiani, D. Ciampini, and E. Arimondo, Phys. Rev. Lett. 87, 140402 (2001).

24. F.S. Cataliotti, S. Burger, C. Fort, P. Maddaloni, F. Minardi, A. Trombettoni, A. Smerzi, and M. Inguscio, Science 293, 843 (2001).

25. M. Greiner, I. Bloch, O. Mandel, T.W. Hänsch, and T. Esslinger, Phys. Rev. Lett. 87, 160405 (2001).

26. M. Greiner, O. Mandel, T. Esslinger, T.W. Hänsch, and I. Bloch, Nature (London) 415, 39 (2002).

27. D.I. Choi and Q. Niu, Phys. Rev. Lett. 82, 2022 (1999).

28. A. Smerzi, A. Trombettoni, P.G. Kevrekidis, and A.R. Bishop, Phys. Rev. Lett. 89, 170402 (2002); F.S. Cataliotti, L. Fallani, F. Ferlaino, C. Fort, P. Maddaloni, M. Inguscio, New J. Phys. 5, 71 (2003).

29. P.G. Kevrekidis and D.J. Frantzeskakis, Mod. Phys. Lett. B 18, 173 (2004).

30. V.A. Brazhnyi and V.V. Konotop, Mod. Phys. Lett. B 18, 627 (2004).

31. G. Herring, P.G. Kevrekidis, R. Carretero-González, B.A. Malomed, D.J. Frantzeskakis, A.R. Bishop. "Trapped bright matter-wave solitons in the presence of localized inhomogeneities", Phys. Lett. A, in press (2005).

32. G. Theocharis, D.J. Frantzeskakis, R. Carretero-González, P.G. Kevrekidis, and B.A. Malomed, Phys. Rev. E 71, $017602(2005)$.

33. P.G. Kevrekidis, D.J. Frantzeskakis, R. Carretero-González, B.A. Malomed, G. Herring, and A.R. Bishop, Phys. Rev. A 71, 023614 (2005).

34. E.P. Gross. Nuovo Cim., 20454 (1961).

35. L.P. Pitaevskii. Sov. Phys. JETP, 13451 (1961).

36. P.A. Ruprecht, M.J. Holland and K. Burnett. Phys. Rev. A, 514704 (1995). 
37. V.M. Pérez-García, H. Michinel and H. Herrero, Phys. Rev. A 57, 3837 (1998);

for a more rigorous derivation of the 1D effective equation, see E.H. Lieb, R. Seiringer, and J. Yngvason, Phys. Rev. Lett. 91, 150401 (2003).

38. Y.B. Band, I. Towers, and B.A. Malomed, Phys. Rev. A 67, 023602 (2003).

39. L. Salasnich, A. Parola and L. Reatto, Phys. Rev. A 65, 043614 (2002).

40. O. Morsch and E. Arimondo, in Dynamics and Thermodynamics of Systems with Long-Range Interactions, T. Dauxois, S. Ruffo, E. Arimondo and S. Wilkens (Eds.) (Springer, Berlin 2002), pp. 312-331.

41. Yu.S. Kivshar and B.A. Malomed, Rev. Mod. Phys. 61, 763 (1989).

42. R. Scharf and A.R. Bishop, Phys. Rev. E 47, 1375 (1993).

43. T. Kapitula, Physica D 156, 186 (2001).

44. V.E. Zakharov and A.B. Shabat, Zh. Eksp. Teor. Fiz. 64, 1627 (1973) [Sov. Phys. JETP 37, 823 (1973)].

45. W.P. Reinhardt and C.W. Clark, J. Phys. B 30, L785 (1997);

Th. Busch and J.R. Anglin, Phys. Rev. Lett. 84, 2298 (2000);

G. Huang, J. Szeftel, and S. Zhu, Phys. Rev. A 65, 053605 (2002);

V.A. Brazhnyi and V.V. Konotop, Phys. Rev. A 68, 043613 (2003).

46. D.J. Frantzeskakis, G. Theocharis, F.K. Diakonos, P. Schmelcher, and Yu.S. Kivshar, Phys. Rev. A 66, 053608 (2002).

47. G. Theocharis, D.J. Frantzeskakis, R. Carretero-González, P.G. Kevrekidis, and B.A. Malomed. "Dark soliton dynamics in spatially inhomogeneous media: Application to Bose-Einstein condensates". In press, Math. Comput. Simulat. (2005).

48. N.G. Parker, N.P. Proukakis, M. Leadbeater, and C.S. Adams, Phys. Rev. Lett. 90, 220401 (2003);

N.G. Parker, N.P. Proukakis, M. Leadbeater, and C.S. Adams, J. Phys. B 36, 2891 (2003);

N. P. Proukakis, N.G. Parker, D.J. Frantzeskakis, and C.S. Adams, J. Opt. B: Quantum Semiclass. Opt. 6, S380 (2004).

49. N.G. Parker, N.P. Proukakis, C.F. Barenghi, and C.S. Adam, J. Phys. B: At. Mol. Opt. Phys. 37, S175 (2004).

50. M. Grillakis, J. Shatah and W. Strauss, J. Funct. Anal. 74, 160 (1987);

ibid. 94, 308 (1990).

51. T. Kapitula, P.G. Kevrekidis and B. Sandstede, Physica D, 195, 263 (2004).

52. D.E. Pelinovsky, Inertial law for spectral stability of solitary waves in coupled nonlinear Schrödinger equations, preprint (2003).

53. Y.-J. Oh, Comm. Math. Phys. 121, 11 (1989);

ibid., J. Diff. Eq. 81, 255 (1989).

54. D.N. Neshev, T.J. Alexander, E.A. Ostrovskaya, Yu.S. Kivshar, H. Martin, I. Makasyuk, and Z. Chen, Phys. Rev. Lett. 92, 123903 (2004).

55. J.W. Fleischer, G. Bartal, O. Cohen, O. Manela, M. Segev, J. Hudock, and D.N. Christodoulides, Phys. Rev. Lett. 92, 123904 (2004). 